\title{
Phylogeny, Plant Species, and Plant Diversity Influence Carbon Use Phenotypes Among Fusarium Populations in the Rhizosphere Microbiome
}

\author{
Nicholas LeBlanc, Mycology \& Nematology Genetic Diversity \& Biology Laboratory, U.S. Department of \\ Agriculture-Agriculture Research Service (USDA-ARS), Beltsville, MD, and Oak Ridge Institute for Science and \\ Education, ARS Research Participation Program, Oak Ridge, TN; Adil Essarioui, National Institute of Agronomic \\ Research, Regional Center of Errachidia, Errachidia, Morocco; Linda Kinkel, Department of Plant Pathology, \\ University of Minnesota, St. Paul, MN; and H. Corby Kistler, ${ }^{\dagger}$ Department of Plant Pathology, University of \\ Minnesota, and Cereal Disease Laboratory, USDA-ARS, St. Paul, MN
}

Accepted for publication 29 September 2017.

\section{ABSTRACT}

Carbon use by microorganisms in the rhizosphere microbiome has been linked to plant pathogen suppression and increased mineralization of soil nutrients for plant uptake; however, factors that influence carbon use traits are poorly understood for most microbial groups. This work characterized the relationships of phylogeny, plant species, and plant diversity with carbon use among fungi in the genus Fusarium from rhizosphere soil. Eightyfour randomly collected Fusarium isolates were cultured from the rhizosphere of the perennial plants Lespedeza capitata and Andropogon gerardii, maintained as long-term monocultures or growing in 16-plant species polycultures. For each isolate, a portion of the RPB2 locus was sequenced for phylogenetic analyses and growth on 95 carbon substrates was measured using Biolog SF-P2 plates. Similarity in carbon use among isolates decreased with increasing genetic distance and there were differences in niche width (i.e., number of carbon substrates used) and growth on preferred substrates (i.e., mean growth on the five carbon substrates supporting the greatest growth) among isolates within two predominant phylogenetic clades. Carbon use also varied with plant species and the diversity of the surrounding plant community. Within each of the two predominant clades, niche width was greater among Fusarium isolates from the rhizosphere of $L$. capitata than $A$. gerardii. The correspondence of phylogeny with carbon use suggests changes in Fusarium community composition may lead to the differential use of carbon substrates in the rhizosphere, while the effects of plant species and diversity suggest variation in plants communities may also correspond to variation in carbon use by these fungi. In addition, the consistent effect of plant species on niche width within different clades provides evidence that the rhizosphere environment of the two plants selects for particular traits, rather than promoting the presence of clades with those traits. Overall, this research shows the dynamics of plant and fungal communities are likely to influence carbon use in the rhizosphere and consequently processes related to this phenotype, such as soil nutrient cycling and competition for carbon among soil microbes.
Soil microbe-mediated feedbacks on plant growth and fitness are recognized as key components of native and agricultural ecosystems (Bever et al. 2010, 2012; Van Nuland et al. 2016). Traditionally, interactions between plants and soil microorganisms (plant-soil feedbacks) have been studied in the context of highly coevolved

${ }^{\dagger}$ Corresponding author: H. Kistler; E-mail: hckist@umn.edu

*The $\boldsymbol{e}$-Xtra logo stands for "electronic extra" and indicates that one supplementary table is published online.

This article is in the public domain and not copyrightable. It may be freely reprinted with customary crediting of the source. The American Phytopathological Society, 2017. symbionts that have clear negative (e.g., pathogens [Liu et al. 2012; Mills and Bever 1998]) or positive feedbacks (e.g., mycorrhizal fungi or rhizobial bacteria [Revillini et al. 2016]) on plant growth. However, the focus on highly coevolved or specific plant-microbe symbiotic interactions ignores the significance of indirect effects of microorganisms on plant growth. Such indirect effects include the ability of free-living microorganisms in the soil or rhizosphere microbiome to change the availability of plant nutrients through decomposition and soil mineralization (Weidner et al. 2015) or suppress the activity of soilborne plant pathogens (Kinkel et al. 2011).

Though many eukaryotic and prokaryotic microorganisms influence soil nutrient cycling, fungi in the genus Fusarium are distinctive considering their high capacity to break down and use a wide variety 
of carbon substrates (King et al. 2011; Li et al. 2008; Regalado et al. 1997). In particular, comparison of Fusarium genomes with genomes of more than 90 other fungi shows an enrichment for genes predicted to produce lignocellulosic enzymes (i.e., CAZymes) in Fusarium genomes (Zhao et al. 2013). These phenotypic and genomic capacities coupled with the cosmopolitan distribution of Fusarium (Burgess 1982) implicates these fungi in the global decomposition and transformation of carbon substrates in soil, potentially increasing mineralization of plant nutrients. In addition to their possible influence on soil carbon and nutrient dynamics, nonpathogenic Fusarium have been suggested to suppress soilborne plant pathogens in the same genus through competition for growth-limiting carbon in soil (Alabouvette et al. 2009; Couteaudier and Alabouvette 1990). These examples highlight two indirect mechanisms through which carbon use by Fusarium may affect plant health and productivity. Determining the factors that influence variation in carbon use among Fusarium communities and populations in the soil and rhizosphere microbiome will refine understanding of the potential impacts of these fungi on plants in agricultural and native ecosystems.

Variation in root exudates among plant species and genotypes is hypothesized to contribute to variation in soil nutrients and the composition of microbial communities in soil (Badri et al. 2009; Broeckling et al. 2008). This variation in plant-derived nutrients may also influence carbon use among heterotrophic soil microorganisms by selection within the rhizosphere. For example, high concentrations of homoserine in the rhizosphere of pea (Pisum sativum) results in selective enrichment of fungi that are able to use this substrate (Rodriguez-Carres et al. 2008). Similarly, Gram-positive bacteria in the genus Streptomyces isolated from the rhizosphere of different plant species exhibit differences in carbon use phenotypes (Essarioui et al. 2016). Variation in the types and diversity of carbon substrates in the rhizosphere of different plant species may also influence carbon use by rhizosphere microorganisms. This is suggested by research showing treatment of microbial populations with greater numbers of carbon substrates selects for an increased capacity to use more substrates by those populations (Gravel et al. 2011).

Plant diversity has varied effects on microbial communities in soil and consistently increases carbon in soil (Lange et al. 2014; Tilman et al. 1997). Increased plant diversity has been linked to greater fungal diversity (LeBlanc et al. 2015; Peay et al. 2013). In contrast, the diversity of prokaryotic communities in soil has shown negative responses (Schlatter et al. 2015) as well as no response (Dassen et al. 2017). Differences in rhizosphere carbon composition among different types of plants (Badri et al. 2009) may also lead to a greater diversity of carbon substrates in soil of diverse polyculture plant communities compared with monoculture plant communities.

The goal of this research was to characterize carbon use among Fusarium isolates from rhizosphere soil to identify potential correlates of variation in carbon use phenotypes. Specifically, we propose to investigate the relationships among plant host, plant community diversity, Fusarium phylogeny, and carbon use phenotypes among Fusarium isolates from the rhizosphere microbiome. Carbon use phenotypes provide fundamental insight into the ecological roles of fungi in phytobiomes and are related to capacities to suppress plant pathogens (Alabouvette et al. 2009; Essarioui et al. 2017) and to nutrient cycling dynamics in soil. We pose three hypotheses in our work. First, we hypothesize that Fusarium isolates from the rhizosphere of different plant species and plant diversity treatments will differ in carbon use phenotypes (niche width, growth efficiency, and growth on preferred substrates). Second, we hypothesize that more closely related Fusarium isolates will display greater similarity in carbon use phenotypes than distantly related fungi. Finally, we hypothesize that, within phylogenetic clades, Fusarium isolates from the same plant species or diversity treatment will have more similar carbon use phenotypes than isolates from different plant species or diversity. Overall, this work provides novel insights into the relationships between Fusarium phylogeny and carbon use, the influences of plant communities on Fusarium carbon use phenotypes, and the potential ecological roles of these fungi as mediators of soil nutrient cycling and or as competitors for growth limiting carbon in the phytobiome.

\section{MATERIALS AND METHODS}

Field sampling and soil sample processing. Soil was sampled from the Cedar Creek Ecosystem Science Reserve, a National Science Foundation LTER site in east-central Minnesota in July 2012. Two soil cores, separated by a $90^{\circ}$ angle around the base of the sampled plant, were taken from the base of individual plants growing in monoculture or 16-species (i.e., polyculture) plant communities as part of replicated experimental plots established and maintained since 1994 (Tilman et al. 1997). The individual plants sampled were the perennial grass Andropogon gerardii and the perennial legume Lespedeza capitata, identified by inflorescence morphology. The two plant species were selected due to their predominance in prairie ecosystems and evolutionary relationship with common cultivated grasses (e.g., Triticum aestivum) and legumes (e.g., Glycine max) in the region. Two soil cores per individual plant sampled, from a total of 12 plants, were pooled in the field and kept on ice until storage at $-20^{\circ} \mathrm{C}$. Extractable phosphorus, extractable potassium, extractable nitrate, $\mathrm{pH}$, and organic matter of the soil samples were measured at the University of Minnesota Research Analytical Laboratory (ral.cfans.umn.edu), as previously reported (Essarioui et al. 2016).

Culturing of Fusarium isolates from soil. To culture Fusarium isolates from the soil samples, Fusarium-selective Komada and NashSnyder media (Leslie and Summerell 2006) were inoculated with soil suspensions, as previously reported (Essarioui et al. 2017). Soil suspensions were made by diluting $1 \mathrm{~g}$ of air-dried soil in sterile water for a final concentration of $1 \times 10^{-3} \mathrm{~g}$ of soil $/ \mathrm{ml}$ of water. For each individual soil sample, $1 \mathrm{ml}$ of the diluted soil suspension was inoculated and spread onto two plates of each type of media. The inoculated plates were incubated at $28^{\circ} \mathrm{C}$ for 5 days. Individual colonies for subculturing were selected randomly using a grid and randomized coordinates. Colonies were subcultured onto $1 / 2$-strength potato dextrose agar (1/2-PDA: $12 \mathrm{~g}$ of potato dextrose broth (Difco) $+15 \mathrm{~g}$ of agar/liter) and grown under a $12 \mathrm{~h}$ photoperiod at $25^{\circ} \mathrm{C}$ for 5 to 7 days. A culture was classified as within the genus Fusarium based on the production of fusiform-shaped (banana or canoe) spores. For individual soil samples failing to produce more than five Fusarium isolates after the initial soil suspension, repeated soil suspensions were performed. Single-spore isolates were generated from the cultured Fusarium isolates and stored in $50 \%$ glycerol at $-70^{\circ} \mathrm{C}$.

Carbon use phenotyping. Carbon use phenotypes were measured based on the ability of the cultured Fusarium isolates to grow on 95 different carbon substrates using Biolog SF-P2 plates (Biolog, Hayward, CA). A portion of these data have previously been reported and the methods used to standardize fungal material and inoculate the Biolog SF-P2 plates have been described in Essarioui et al. (2017). Briefly, individual isolates were grown for 5 days on 1/2-PDA at $25^{\circ} \mathrm{C}$ under a $12 \mathrm{~h}$ photoperiod. Spores from each isolate were harvested from the media using $0.2 \%$ carrageenan in sterile water and spore suspensions were adjusted to an optical density (OD) of 0.22 at $590 \mathrm{~nm}$. One and a half milliliter of the adjusted spore suspensions was dispensed into $13.5 \mathrm{ml}$ of sterile water. The spore suspensions for each isolate were then inoculated into individual Biolog SF-P2 plates (i.e., one isolate per plate). Optical densities within the 96 wells were measured 96 h (4 days) postinoculation using a Synergy H1 Reader (BioTek Instruments, Winooski, VT) at $590 \mathrm{~nm}$. 
Processing of the Biolog data and focus on specific of carbon use traits followed a similar rationale outlined by previous work using similar methods (Essarioui et al. 2016; Schlatter et al. 2013). Optical densities measured from the water control well of each plate were subtracted from OD of all the 95 remaining wells, after which individual wells with $\mathrm{OD} \leq 0.005$ were set to 0 . Three main carbon use traits were calculated from the processed OD values representing growth of individual isolates on the 95 carbon substrates. The three traits were represented the number of substrates supporting growth (niche width), the capacity to produce biomass across the used substrates (growth efficiency), and the capacity to produce biomass on the preferred substrates (growth on preferred substrates). Niche width was calculated as the number of carbon substrates yielding positive OD values greater than 0.005 for a given isolate. Growth efficiency was calculated as the mean OD across the 95 carbon substrates for a given isolate. Growth on preferred substrates was calculated as the mean OD across the five substrates with the highest OD values.

PCR amplification and Sanger sequencing. A portion of the $R P B 2$ (DNA directed RNA polymerase second largest subunit) gene was amplified and sequenced from each Fusarium isolate to measure the phylogenetic relatedness among and genetic distance between the isolates. These data were also used for preliminary taxonomic classification. Deoxyribonucleic acids were extracted using a cetrimonium bromide (CTAB)/chloroform-based protocol as described in Gale et al. (2011) and diluted to $10 \mathrm{ng} / \mu \mathrm{l}$. A portion of the $R P B 2$ gene was amplified from the extracted DNA using the RPB2-5F2 and RPB2-7cR primers (O'Donnell et al. 2007). Reactions were performed in an Eppendorf Mastercycler (Eppendorf, Hamburg, Germany) using 20- $\mu$ l reactions with $2 \mu$ l of Ex-Taq buffer, $1.6 \mu \mathrm{l}$ of dNTPs (2.5 $\mu \mathrm{M}$ of each), $2 \mu \mathrm{l}$ of each forward and reverse primers $(10 \mu \mathrm{M}), 11.3 \mu \mathrm{l}$ of water, $0.1 \mu \mathrm{l}$ of Takara Ex-Taq (Takara Bio Inc., Shiga, Japan) polymerase (5 units/ $\mu 1$ ), and $1 \mu \mathrm{l}$ template (10 ng of DNA/ $\mu \mathrm{l})$. The PCR reactions were performed with an initial denaturing step at $95^{\circ} \mathrm{C}(5 \mathrm{~min})$, followed by 24 cycles at $95^{\circ} \mathrm{C}(1 \mathrm{~min}), 57^{\circ} \mathrm{C}(1 \mathrm{~min}), 72^{\circ} \mathrm{C}(1 \mathrm{~min})$, and ended with a final extension of $72^{\circ} \mathrm{C}(10 \mathrm{~min})$. Amplicons were purified using a Promega Wizard kit (Promega, Madison, WI) and sequenced with the RPB2-5F2 primer using Sanger technology at the Biomedical Genomics Center at the University of Minnesota (http://genomics. umn.edu). Five prime and $3^{\prime}$ low quality sequence data were trimmed from the sequences using 4Peaks (http://nucleobytes.com/4peaks/). Processed sequence data were aligned using MUSCLE (Edgar 2004). Sequences have been deposited in as GenBank accession numbers KY354597 to KY354680.

Phylogenetics and taxonomy. To determine the phylogenetic relatedness among the cultured Fusarium isolates, the aligned RPB2 sequences were used to construct a phylogenetic tree using the neighbor-joining method implemented in MEGA6 (Tamura et al. 2013). Clade support was measured based on nonparametric bootstrap values, with 100 replications. For taxonomic identification of the isolates, the same sequence data were aligned to culture-backed $R P B 2$ reference sequences in the Fusarium ID database (Geiser et al. 2004) using BLASTN (Altschul et al. 1997).

Statistics. All statistics were performed in the R environment (R Development Core Team 2015), using the corrplot (Wei 2013), ggplot2 (Wickham 2009), seqinr (Charif and Lobry 2007), and vegan (Oksanen et al. 2016) packages.

A Mantel test was used to test for a relationship between genetic and carbon use distance. To perform the Mantel test, a genetic distance (square root of the pairwise sequence identity) matrix was calculated from the aligned $R P B 2$ sequence data using the seqinr package and a carbon use distance (Euclidean) matrix was calculated from OD values from growth of individual isolates on the 95 distinct carbon substrates. The Mantel test was performed with the vegan package using the Pearson correlation method with 999 permutations.

To visualize pairwise correlation in growth among Fusarium isolates across the 95 carbon substrates, a heat-map was generated with the corrplot package (Wei 2013). Pearson correlation coefficients were calculated from OD values from growth of individual isolates on the 95 carbon substrates for a given comparison between pairwise isolates. The isolates represented in the heat-map were reordered to match the order of the isolates in the phylogeny constructed from the RPB2 sequence data (see above).

Two-sided $t$ tests were used to test for differences in the three carbon use traits (described above) between two main phylogenetic clades as well as for differences between isolates from $A$. gerardii versus $L$. capitata or monoculture versus polyculture, within the clades. Two-sided $t$ tests with false discover rate (FDR) (Benjamini and Hochberg 1995) correction for multiple comparisons were used to test for differences in growth on the 95 individual substrates between isolates from different phylogenetic clades, different plant species, and different levels of plant diversity. Two-way analysis of variance tests (two-way ANOVA) were used to test the effect of plant species, plant diversity, and the interaction between plant species and diversity on the three carbon use traits. Finally, Spearman rank correlation coefficients $(\rho)$ were used to test for relationships between niche width, growth efficiency, and growth on preferred substrates with individual soil edaphic characteristics, including organic matter, potassium, $\mathrm{pH}$, phosphorus, and nitrogen.

\section{RESULTS}

Variation in recovered isolates and carbon use. A total of 84 Fusarium isolates were cultured from the 12 individual soil samples. There was variation in the number of isolates cultured from the individual soil samples. Within soil from the different plant species, a mean of $4.33(\sigma=2.944, \min =2, \max =10)$ isolates were cultured from the rhizosphere of individual A. gerardii plants and a mean of $9.667(\sigma=0.816, \min =8, \max =10)$ isolates were cultured from the rhizosphere of each L. capitata plant. Within soil from the different plant diversity treatments, a mean of $6.333(\sigma=3.445$, $\min =2$, $\max =10)$ isolates were cultured from monoculture and a mean of $7.667(\sigma=3.67, \min =2, \max =10)$ isolates were cultured from polyculture plots.

All the 95 carbon substrates were used by at least one Fusarium isolate. The mean number of substrates used by each isolate was $91.31(\sigma=3.818, \min =74, \max =95)$. Among the 84 Fusarium isolates there were 56 unique carbon use phenotypes (i.e., combinations of carbon substrates used by individual isolates). Fifty of these patterns were unique to a single isolate, while the most common carbon use phenotype (use of all 95 carbon substrates) was observed for 14 isolates. The five substrates used by all of the isolates and supporting the greatest growth (i.e., highest mean OD among isolates) were turanose, $N$-acetyl-D-glucoseamine, polyoxyethylene (20) sorbitan monopalmitate (Tween 40), D-trehalose, and polyoxyethylene (20) sorbitan monooleate (Tween 80). The five substrates used the least frequently and also supporting the least growth were 3 '-methyl-Dglucose, adenosine, adenosine-5'-monophosphate, D-fructose-6phosphate, and D-L- $\alpha$-glycerol phosphate.

Taxonomy and phylogeny of Fusarium isolates. Taxonomic classification of the isolates was based on alignment and similarity of $R P B 2$ sequence data from the isolates with $R P B 2$ reference sequences in the Fusarium ID database (Geiser et al. 2004). Based on the alignments, the $R P B 2$ sequences from the 84 isolates were similar to seven different culture-backed reference sequences, representing six different phylogenetic species: F. oxysporum, F. foetens, F. commune, $F$. brachygibbosum, $F$. incarnatum-equiseti, and $F$. solani (Table 1). 
A neighbor-joining phylogeny was constructed using RPB2 sequence data to assess the phylogenetic relatedness among the isolates and the presence of different phylogenetic clades. The Fusarium

TABLE 1

Taxonomic classification of the Fusarium isolates

\begin{tabular}{|c|c|c|}
\hline Reference $^{\mathrm{a}}$ & Isolates ${ }^{\mathrm{b}}$ & $\begin{array}{c}\text { Sequence } \\
\text { identity }^{\mathrm{C}}\end{array}$ \\
\hline F. oxysporum NRRL34936 & 35 & 0.995 \\
\hline F. foetens NRRL38302 & 24 & 0.99 \\
\hline F. commune NRRL28387 & 6 & 0.979 \\
\hline F. brachygibbosum NRRL31008 & 1 & 1 \\
\hline $\begin{array}{l}\text { F. incarnatum-equiseti 14-b } \\
\text { NRRL20697 }\end{array}$ & 1 & 0.985 \\
\hline F. solani 5-d NRRL43681 & 14 & 1 \\
\hline F. solani 5-I NRRL32791 & 3 & 1 \\
\hline \multicolumn{3}{|c|}{$\begin{array}{l}\text { a Species and strain designation of the references in Fusarium ID } \\
\text { (Geiser et al. 2004) that were most similar to the RPB2 sequences } \\
\text { from the Fusarium isolates. } \\
\text { b Total number of Fusarium isolates for which RPB2 sequence data } \\
\text { aligned (BLASTN) to references shown in column } 1 \text {. } \\
\text { c Mean identity between RPB2 sequences of the isolates and RPB2 } \\
\text { sequences of the references shown in column } 1 \text {. }\end{array}$} \\
\hline
\end{tabular}

isolates formed four different phylogenetic clades supported by bootstrap values of at least $97 \%$ (Fig. 1A). The previous taxonomic classification of the isolates showed that the largest clade (59 of 84 isolates) represented isolates showing high $R P B 2$ sequence similarity to $F$. oxysporum reference sequences and the closely related phylogenetic species $F$. foetens. Because distinguishing between $F$. oxysporum and $F$. foetens based on the length of the isolate $R P B 2$ sequence data are not feasible, this clade will be referred to as $F$. oxysporum, hereafter. The second largest clade (17 of 84 isolates) represented isolates showing high sequence similarity to $F$. solani reference sequences (referred to as the $F$. solani clade, hereafter) (Fig. 1A).

Phylogeny and carbon use of the Fusarium isolates. Similarity in carbon use among the Fusarium isolates was greater within than between phylogenetic clades. As shown in the heat-map, Pearson correlation coefficients were generally higher for isolate pairs within phylogenetic clades than between phylogenetic clades (Fig. 1A and B). There was also a significant positive relationship between genetic distance and carbon use distance (Mantel correlation $=0.294, P<0.01$ ).

Carbon use traits also differed between the two largest phylogenetic clades, isolates closely related to $F$. oxysporum and $F$. solani (Table 1; Fig. 1A). Niche width (the number of substrates used) was greater for isolates in the $F$. oxysporum clade compared with isolates in the $F$. solani clade (two-sided $t$ test, $P<0.01$ ). In contrast, growth on preferred substrates (mean OD across the five substrates with the highest OD for every isolate) was greater for
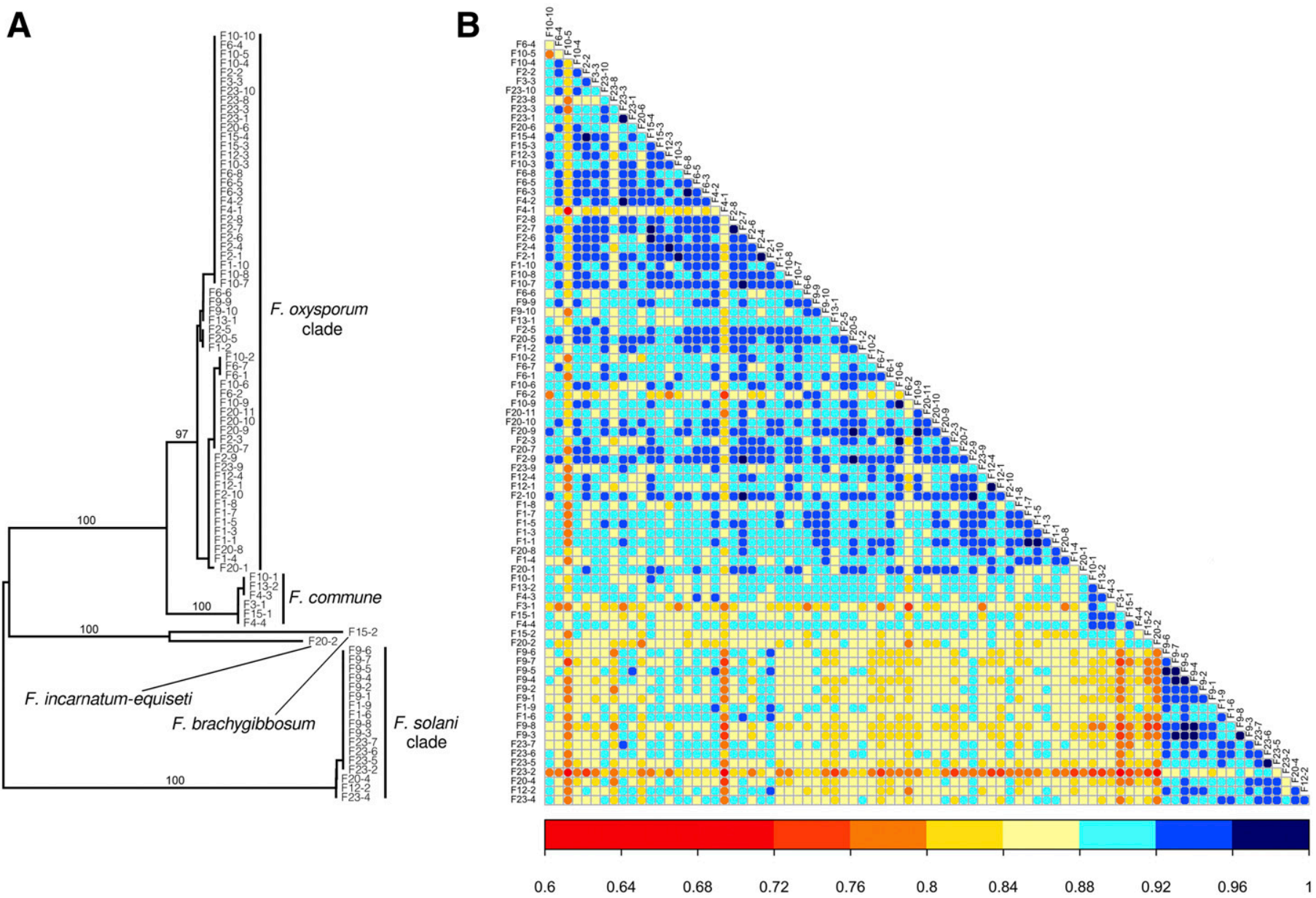

Fig. 1. A, A neighbor-joining phylogeny representing phylogenetic relationships between Fusarium isolates and B, a heat-map representing similarity in carbon use among isolates. The phylogeny was constructed from $R P B 2$ sequence data from the isolates and numbers above branches represent nonparametric bootstrap values. Taxonomy is based on alignment of RPB2 sequence data to Fusarium ID (Geiser et al. 2004) (Table 1). F. oxysporumand F. solani clades represent the two clades used in additional analyses. The heat-map represents Pearson correlation coefficients calculated from optical density (OD) values (growth) on the 95 carbon substrates for a given comparison between pairwise isolates. The isolates represented in the heat-map were reordered to match the order of the isolates represented in the phylogeny. 
isolates in the $F$. solani clade than isolates in the $F$. oxysporum clade (two-sided $t$ test, $P<0.01$ ). Growth on individual substrates also differed between these two clades. Following two-sided $t$ tests and FDR based correction for multiple comparisons, 59 of the 95 substrates supported differential growth of isolates from the two different clades. Members of the $F$. oxysporum clade grew more on 28 of the 59 differentially used substrates (Supplementary Table S1).

Effect of plant species and plant diversity on Fusarium carbon use. Carbon use differed between Fusarium isolates from the rhizosphere of $L$. capitata and A. gerardii, though the differences were dependent on the diversity of the surrounding plant community. Fusarium niche width was significantly affected by plant species (twoway ANOVA, $P<0.001$ ); however, there was also a significant interaction between plant species and plant diversity (two-way ANOVA, $P=0.016$ ). Posthoc comparison of niche width between the plots showed Fusarium isolates from the rhizosphere of $A$. gerardii in polyculture had reduced niche width compared isolates from A. gerardii in monoculture or from $L$. capitata growing in monoculture or polyculture (Tukey's honest significant difference test, $P<0.05$ ) (Fig. 2). Though growth efficiency was not influenced by plant diversity or plant species (data not shown), growth on preferred substrates was. Overall there was a significant effect of plant species (two-way ANOVA, $P=0.013$ ) and plant diversity (twoway ANOVA, $P=0.048$ ) on growth on preferred substrates of the Fusarium isolates. Posthoc comparison showed isolates from the rhizosphere of $A$. gerardii growing in polyculture had significantly greater growth on preferred substrates compared with isolates from the rhizosphere of $L$. capitata growing in monoculture (Tukey's honest significant difference test, $P<0.05$ ) (Fig. 3).

Fusarium isolates from the rhizosphere of L. capitata and A. gerardii, across plant diversity treatments, displayed growth differences on individual carbon substrates. Following correction

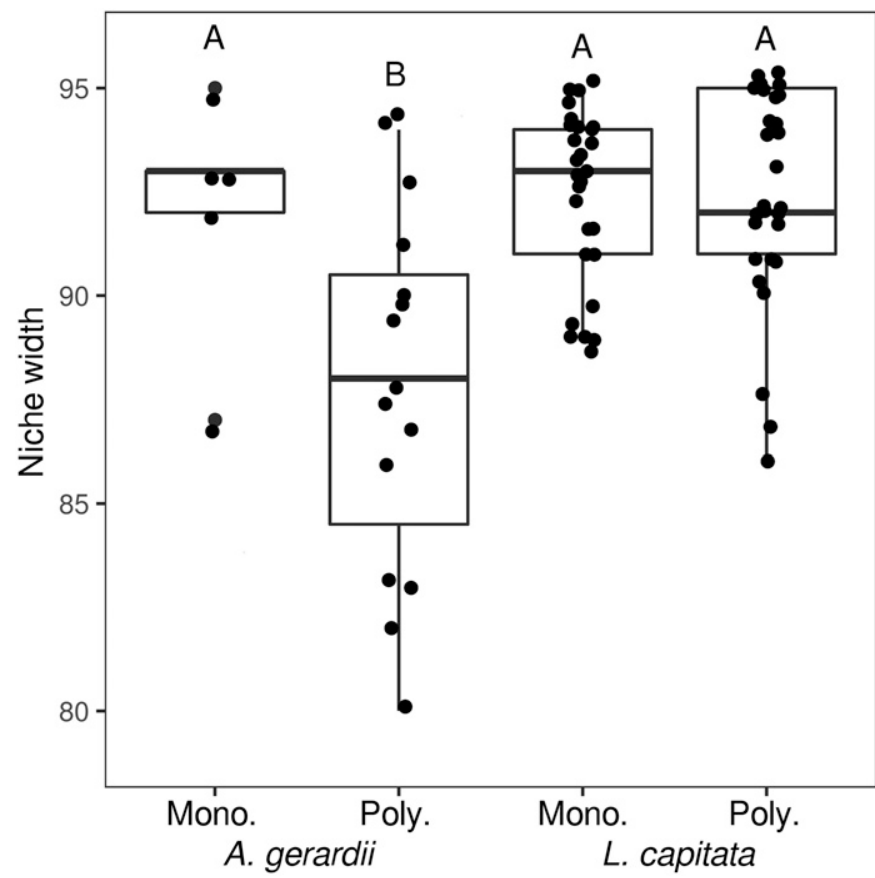

Fig. 2. Box-plots and data representing niche width (number of substrates used) of Fusarium isolates from the rhizosphere of the plant species Andropogon gerardii and Lespedeza capitata, maintained as monocultures (mono.) or growing in polyculture (poly.) plant communities. Different numbers above the box-plots indicate significant $(P<0.05)$ differences between the groups, based on Tukey's honest significant difference posthoc comparisons. for multiple comparisons, 15 of the 95 substrates supported differential growth of isolates from the two plant species. Isolates from A. gerardii grew more on 4 of the 15 differentially used substrates. There were no differences in growth on individual substrates for isolates from monoculture or polyculture plant communities evaluated across plant species (data not shown).

Within the two predominant phylogenetic clades, plant species had a significant effect on the niche width of the Fusarium isolates. Niche width was greater for isolates from the rhizosphere of $L$. capitata than $A$. gerardii for both the $F$. oxysporum (two-sided $t$ test, $P<0.01$; (Fig. 4A) and $F$. solani (two-sided $t$ test, $P<0.001$ ) (Fig. 4B) clades (ANOVA tests used above were not feasible do to the reduced sample sizes within the two clades). Though carbon use differed between these clades (see above), these results show different plant species can have a similar effect on carbon use phenotypes within different phylogenetic clades.

Soil edaphic characteristics and carbon use. Differences in soil edaphic characteristics between soil from the two plant species and levels of plant diversity have been reported previously (Essarioui et al. 2016). As shown in Table 2, save for the positive correlation between Fusarium niche width and soil nitrogen, increases in soil edaphic characteristics corresponded to decreases in niche width, growth efficiency, and growth on preferred substrates. This highlights the potential for variation in the abiotic component of soil to explain variation in Fusarium carbon use.

\section{DISCUSSION}

Carbon use by fungi in the genus Fusarium has implications for their role in decomposition and soil nutrient cycling as well as their ability to suppress soilborne plant pathogens through competition for nutrients. This research characterized the phylogenetic diversity

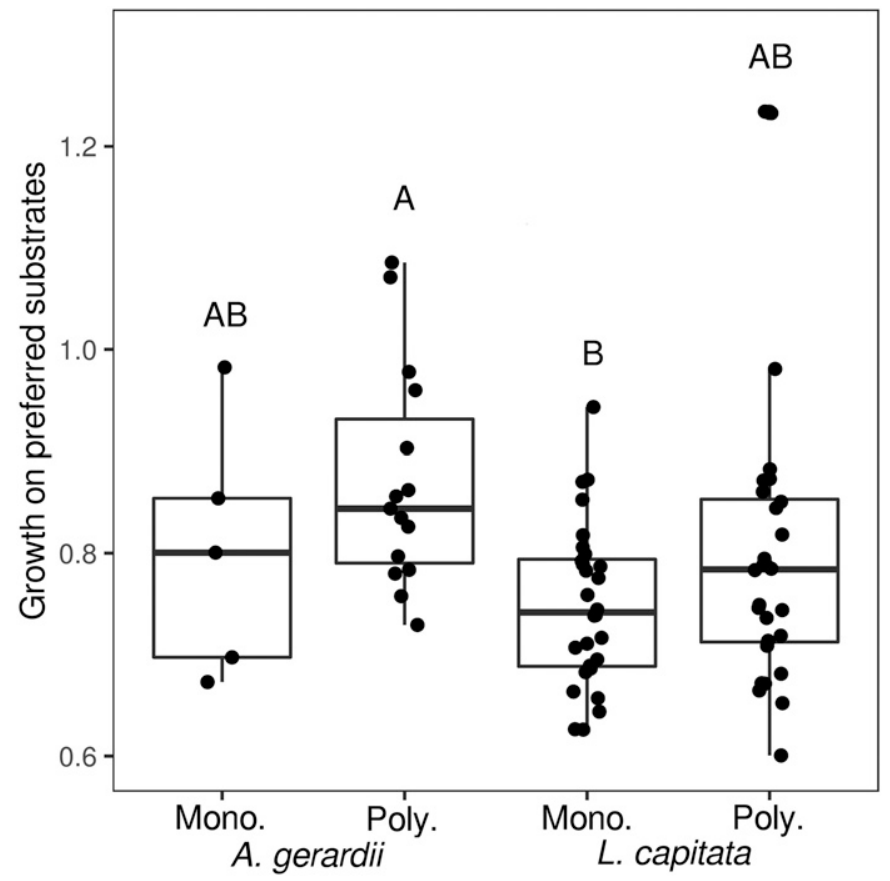

Fig. 3. Box-plots and data representing growth on preferred substrates (mean optical density [OD] across the five substrates with the highest OD) of Fusarium isolates from the rhizosphere of the plant species Andropogon gerardii and Lespedeza capitata, maintained as monocultures (mono.) or growing in polyculture (poly.) plant communities. Different numbers above the box-plots indicate significant $(P<0.05)$ differences between the groups, based on Tukey's honest significant difference posthoc comparisons. 
and carbon use of Fusarium isolates from the rhizosphere of two plant species growing as long-term monocultures or within polymeasurement of Fusarium carbon use under the influence of different plant species or different levels of plant diversity allowed us to determine the importance of evolutionary constraints and ecological context in determining this functionally relevant phenotype. We found Fusarium isolates within phylogenetic clades displayed greater similarity in carbon use than between clades, independent of plant species or diversity. Carbon use differed between isolates from A. gerardii and L. capitata, depending on the diversity of the surrounding plant community. Within different phylogenetic clades, isolates from $A$. gerardii had reduced niche width compared with isolates from $L$. capitata. Overall, this work suggests changes in the composition of Fusarium and plant communities may change Fusarium mediated soil nutrient availability with potential feedbacks on plant growth and the suppression of soilborne plant pathogens.

The cultured Fusarium isolates displayed a high capacity to grow on the 95 assayed carbon substrates; the most common carbon use phenotype was growth on all the 95 carbon sources. Growth across the 95 substrates was very similar among the individual Fusarium isolates (i.e., high positive correlation coefficients). Despite this similarity in carbon use among isolates, there was significant phylogenetic signal in carbon use. With increasing genetic distance among the isolates there was also an increasing dissimilarity in growth on the 95 carbon substrates. Additionally, carbon use was significantly different between isolates within the predominant $F$. oxysporum and $F$. solani clades. This supports our initial hypothesis that carbon use would vary with phylogeny, providing a tentative link between Fusarium community composition and function. Based on empirical study, there is a link between carbon use and the outcome of competition between fungi in soil (Couteaudier and Alabouvette 1990; Rodriguez-Carres et al. 2008). This suggests the variation in the presence or abundance of fungi in these different clades, with different carbon use phenotypes, may influence competitive interactions between Fusarium and other heterotrophic microorganisms. Ultimately, the consequences of differences in Fusarium carbon use exhibited between different phylogenetic clades will be understood by linking specific phenotypes to effects on carbon and nutrient concentrations in soil. culture plant communities. Integrating phylogenetic data with

The differences in niche width and preferred growth of isolates from the rhizosphere of the two plant species growing in different plant diversity treatments provides some support for our initial hypothesis of an effect of plant species and diversity on Fusarium carbon use. However, the effects of plant diversity on niche width was not consistent between plant species. Specifically, Fusarium niche width was greater in the rhizosphere of $A$. gerardii growing in monoculture than the same species in diverse plant communities, while there was no difference in Fusarium niche width in the rhizosphere of $L$. capitata between the two plant diversity treatments. This makes it difficult to generalize the effects of plant species and diversity on Fusarium carbon use in the rhizosphere microbiome. However, these results share similarities with research showing individual plant species do not display consistent changes

\begin{tabular}{|c|c|c|}
\hline \multicolumn{3}{|c|}{$\begin{array}{c}\text { TABLE } 2 \\
\begin{array}{c}\text { Correlation between Fusarium carbon use traits } \\
\text { and soil edaphic characteristics }\end{array}\end{array}$} \\
\hline Carbon use trait $^{\mathrm{a}}$ & $\begin{array}{l}\text { Soil edaphic } \\
\text { characteristic }^{\mathrm{b}}\end{array}$ & $\begin{array}{c}\text { Spearman rho } \\
(P \text { value })^{\mathrm{c}}\end{array}$ \\
\hline Niche width & Nitrogen & $0.312(0.016)$ \\
\hline Niche width & $\mathrm{pH}$ & $-0.509(<0.001)$ \\
\hline Niche width & Potassium & $-0.413(<0.01)$ \\
\hline Niche width & Organic matter & $-0.366(<0.01)$ \\
\hline Growth efficiency & $\mathrm{pH}$ & $-0.368(<0.01)$ \\
\hline Growth efficiency & Potassium & $-0.385(<0.01)$ \\
\hline $\begin{array}{l}\text { Growth on preferred } \\
\text { substrates }\end{array}$ & $\mathrm{pH}$ & $-0.266(0.041)$ \\
\hline $\begin{array}{l}\text { Growth on preferred } \\
\text { substrates }\end{array}$ & Potassium & $-0.284(0.029)$ \\
\hline \multicolumn{3}{|c|}{$\begin{array}{l}\text { a Carbon use trait of Fusarium isolates. See Carbon use phenotyping } \\
\text { section for definitions. } \\
\text { b Edaphic characteristic of soil sample from which the Fusarium } \\
\text { isolates were cultured. } \\
\text { c Spearman rank correlation coefficients and associated } P \text { value } \\
\text { testing the correlation between Fusarium carbon use trait in column } 1 \\
\text { and soil edaphic characteristics in column } 2 \text {. }\end{array}$} \\
\hline
\end{tabular}
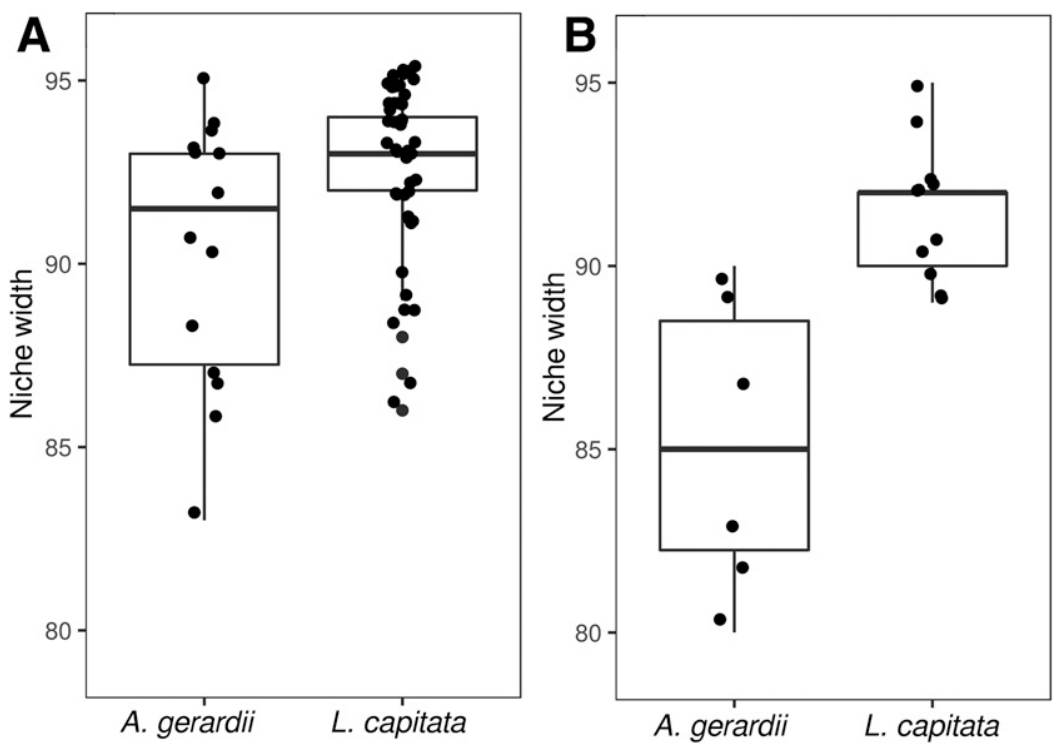

Fig. 4. Box-plots and data representing niche width (number of substrates used) of Fusarium isolates from the rhizosphere of the plant species Andropogon gerardii and Lespedeza capitata, across plant diversity treatments. Based on two-sided $t$ tests, in both the $F$. oxysporum $(\mathbf{A}, P<0.01)$ and $F$. solani (B, $P<0.001$ ) clades from $L$. capitata have greater niche width than isolates from $A$. gerardii. 
in growth (Marquard et al. 2009) or metabolism (Scherling et al. 2010) when diversity of the surrounding plant community is altered. Regardless of the context-specific nature of these results, they do provide a link between the dynamics of plant communities and functional traits within a ubiquitous group of fungi.

With evidence that Fusarium carbon use is influenced by phylogeny the effects of plant species and diversity could simply reflect environmental filtering (Saunders et al. 2010), with these treatments selecting for different carbon use traits restricted to different phylogenetic clades. However, we found a consistent effect of plant species within the rhizosphere of the two plant species. Fusarium isolates within the predominant $F$. oxysporum and $F$. solani clades both displayed greater niche widths in the rhizosphere of L. capitata than A. gerardii. This shows that differences between the two plant species directly influence carbon use of Fusarium in the rhizosphere, rather than simply excluding certain clades based on clade-specific traits (i.e., environmental filtering). Interestingly, parallel research found carbon use niche overlap of these Fusarium isolates with Streptomyces bacteria in the same soil samples was greater in the rhizosphere of $L$. capitata than A. gerardii. While this does not provide a mechanism responsible for the increased niche width of the Fusarium isolates, it does suggest competition for carbon between these two microbial groups will be greater in the rhizosphere of L. capitata than A. gerardii (Essarioui et al. 2017).

This work showed that the use of carbon in the rhizosphere microbiome by Fusarium is determined by the phylogenetic history of the fungi as well as the composition and diversity of the plant communities they are associated with. Linking carbon use and phylogenetics of these fungi suggests that changes in Fusarium community composition may have functional consequences for nutrient mineralization and the growth of other heterotrophic microorganisms, with implications for plant growth. Similarly, the effect of plant species and plant diversity on Fusarium carbon use may also have functional consequences for nutrient cycling dynamics in soil. Future research on the effect of varying carbon use on nutrient cycling and microbiome dynamics will enable the potential for management of Fusarium communities to promote a healthy phytobiome. Finally, though the genus Fusarium is an important soilborne plant pathogen (Blomme et al. 2013; Michielse and Rep 2009), the predominance of nonpathogenic forms in the rhizosphere of healthy plants (Demers et al. 2015; Edel et al. 1997; Nel et al. 2006; Yli-Mattila et al. 2010) raises question as to the broader relevance of these fungi to plant fitness (Steinberg et al. 2016). In addition to their role as saprotrophs explored here, there is growing evidence that the production of secondary metabolites, commonly studied as mycotoxins, can also influence the growth of other soil microbes (van Rij et al. 2005) and plants themselves (Tsavkelova et al. 2012). A broader understanding of these fungi is needed to shed light on their relevance to plant growth in native and agricultural ecosystems, including their roles in nutrient cycling, plant growth promotion, and pathogen suppression as a counterweight to the historical emphasis on plant pathogenicity within this genus.

\section{ACKNOWLEDGMENTS}

We thank D. Tilman and the staff at the Cedar Creek Ecosystem Science Reserve. Research was supported by Agricultural and Food Research Grant Initiative Competitive Grant 2011-67019-30200 from the USDA National Institute of Food and Agriculture.

\section{LITERATURE CITED}

Alabouvette, C., Olivain, C., Migheli, Q., and Steinberg, C. 2009.

Microbiological control of soil-borne phytopathogenic fungi with special emphasis on wilt-inducing Fusarium oxysporum. New Phytol. 184:529-544.
Altschul, S. F., Madden, T. L., Schäffer, A. A., Zhang, J., Zhang, Z., Miller, W., and Lipman, D. J. 1997. Gapped BLAST and PSI-BLAST: A new generation of protein database search programs. Nucleic Acids Res. 25:3389-3402.

Badri, D. V., Quintana, N., Kassis, E. G. E., Kim, H. K., Choi, Y. H., Sugiyama, A., Verpoorte, R., Martinoia, E., Manter, D. K., and Vivanco, J. M. 2009. An ABC transporter mutation alters root exudation of phytochemicals that provoke an overhaul of natural soil microbiota. Plant Physiol. 151:2006-2017.

Benjamini, Y., and Hochberg, Y. 1995. Controlling the false discovery rate: A practical and powerful approach to multiple testing. J. Roy. Stat. Soc. B 57:289-300.

Bever, J., Platt, T. G., and Morton, E. R. 2012. Microbial population and community dynamics on plant roots and their feedbacks on plant communities. Annu. Rev. Microbiol. 66:265-283.

Bever, J. D., Dickie, I. A., Facelli, E., Facelli, J. M., Klironomos, J., Moora, M., Rillig, M. C., Stock, W. D., Tibbett, M., and Zobel, M. 2010. Rooting theories of plant community ecology in microbial interactions. Trends Ecol. Evol. 25: 468-478.

Blomme, G., Ploetz, R., Jones, D., De Langhe, E., Price, N., Gold, C., Geering, A., Viljoen, A., Karamura, D., Pillay, M., Tinzaara, W., Teycheney, P.-Y., Lepoint, P., Karamura, E., and Buddenhagen, I. 2013. A historical overview of the appearance and spread of Musa pests and pathogens on the African continent: Highlighting the importance of clean Musa planting materials and quarantine measures. Ann. Appl. Biol. 162:4-26.

Broeckling, C. D., Broz, A. K., Bergelson, J., Manter, D. K., and Vivanco, J. M. 2008. Root exudates regulate soil fungal community composition and diversity. Appl. Environ. Microbiol. 74:738-744.

Burgess, L. W. 1982. General ecology of the fusaria. In: Fusarium: Diseases, Biology, and Taxonomy. P. E. Nelson, T. A. Toussoun, and R. J. Cook, eds. The Pennsylvania State University Press, PA.

Charif, D., and Lobry, J. R. 2007. SeqinR 1.0-2: A contributed package to the R Project for Statistical Computing devoted to biological sequences retrieval and analysis. Pages 207-232 in: Structural Approaches to Sequence Evolution: Molecules, Networks, Populations. U. Bastolla, M. Porto, H. E. Roman, and M. Vendruscolo, eds. Springer-Verlag, New York.

Couteaudier, Y., and Alabouvette, C. 1990. Quantitative comparison of Fusarium oxysporum competitiveness in relation to carbon utilization. FEMS Microbiol. Ecol. 74:261-267.

Dassen, S., Cortois, R., Martens, H., de Hollander, M., Kowalchuck, G. A., van der Putten, W. H., and De Deyn, G. B. 2017. Differential responses of soil bacteria, fungi, archaea and protists to plant species richness and plant functional group identity. Mol. Ecol. 26:4085-4098.

Demers, J. E., Gugino, B. K., and Jiménez-Gasco, M. M. 2015. Highly diverse endophytic and soil Fusarium oxysporum populations associated with fieldgrown tomato plants. Appl. Environ. Microbiol. 81:81-90.

Edel, V., Steinberg, C., Gautheron, N., and Alabouvette, C. 1997. Populations of non-pathogenic Fusarium oxysporum associated with roots of four plant species compared to soilborne populations. Phytopathology 87:693-697.

Edgar, R. C. 2004. MUSCLE: Multiple sequence alignment with high accuracy and high throughput. Nucleic Acids Res. 32:1792-1797.

Essarioui, A., Kistler, H. C., and Kinkel, L. 2016. Nutrient use preferences among soil Streptomyces suggest greater resource competition in monoculture than polyculture plant communities. Plant Soil 409:329-343.

Essarioui, A., LeBlanc, N., Kistler, H. C., and Kinkel, L. 2017. Plant community richness mediates inhibitory interactions and resource competition between Streptomyces and Fusarium populations in the rhizosphere. Microbiol. Ecol. 74:157-167.

Gale, L. R., Harrison, S. A., Ward, T. J., O’Donnell, K., Milus, E. A., Gale, S. W., and Kistler, H. C. 2011. Nivalenol-type populations of Fusarium graminearum and $F$. asiaticum are prevalent on wheat in southern Louisiana. Phytopathology 101:124-134.

Geiser, D. M., Jiménez-Gasco, M. M., Kang, S., Makalowska, I., Veeraraghavan, N., Ward, T. J., Zhang, N., Kuldau, G. A., and O'Donnell, K. 2004. FUSARIUM-ID v. 1.0: A DNA sequence database for identifying Fusarium. Eur. J. Plant Pathol. 110:473-479.

Gravel, D., Bell, T., Barbera, C., Bouvier, T., Pommier, T., Venail, P., and Mouquet, N. 2011. Experimental niche evolution alters the strength of the diversity-productivity relationship. Nature 469:89-92.

King, B. C., Waxman, K. D., Nenni, N. V., Walker, L. P., Bergstrom, G. C., and Gibson, D. M. 2011. Arsenal of plant cell wall degrading enzymes reflects host preference among plant pathogenic fungi. Biotechnol. Biofuels 4:4.

Kinkel, L., Bakker, M. G., and Schlatter, D. C. 2011. A coevolutionary framework for managing disease suppressive soils. Annu. Rev. Phytopathol. 49:47-67.

Lange, M., Eisenhauer, N., Sierra, C. A., Bessler, H., Engels, C., Griffiths, R. I., Mellado-Vázquez, P. G., Malik, A. A., Roy, J., Scheu, S., Steinbeiss, S., 
Thomson, B. C., Trumbore, S. E., and Gleixner, G. 2014. Plant diversity increases soil microbial activity and soil carbon storage. Nat. Commun. 6:1-8.

LeBlanc, N., Kinkel, L., and Kistler, H. C. 2015. Soil fungal communities respond to grassland plant community richness and soil edaphics. Microbiol. Ecol. 70:188-195.

Leslie, J. F., and Summerell, B. A. 2006. The Fusarium Laboratory Manual. Blackwell, Ames, IA.

Li, L., Li, X.-Z., Tang, W.-Z., Zhao, J., and Qu, Y.-B. 2008. Screening of a fungus capable of powerful and selective delignification on wheat straw. Lett. Appl. Microbiol. 47:415-420.

Liu, Y., Yu, S., Xie, Z.-P., and Staehelin, C. 2012. Analysis of a negative plantsoil feedback in a subtropical monsoon forest. J. Ecol. 100:1019-1028.

Marquard, E., Weigelt, A., Roscher, C., Gubsch, M., Lipowsky, A., and Schmid, B. 2009. Positive biodiversity-productivity relationship due to increased plant diversity. J. Ecol. 97:696-704.

Michielse, C. B., and Rep, M. 2009. Pathogen profile update: Fusarium oxysporum. Mol. Plant Pathol. 10:311-324.

Mills, K. E., and Bever, J. D. 1998. Maintenance of diversity within plant communities: Soil pathogens as agents of negative feedback. Ecology 79: 1595-1601.

Nel, B., Steinberg, C., Labuschagne, N., and Viljoen, A. 2006. Isolation and characterization of nonpathogenic Fusarium oxysporum isolates from the rhizosphere of healthy banana plants. Plant Pathol. 55:207-216.

O’Donnell, K., Sarver, B. A. J., Brandt, M., Chang, D. C., Noble-Wang, J., Park, B. J., Sutton, D. A., Benjamin, L., Lindsley, M., Padhye, A., Geiser, D. M., and Ward, T. J. 2007. Phylogenetic diversity and microsphere arraybased genotyping of human pathogenic fusaria, including isolates from the multistate contact lens-associated U.S. keratitis outbreaks of 2005 and 2006. J. Clin. Microbiol. 45:2235-2248.

Oksanen, J., Blanchet, F. G., Friendly, M., Kindt, R., Legendre, P., McGlinn, D., Minchin, P., O’Hara, R. B., Simpson, G. L., Solymos, P., Stevens, M. H. H., Szoecs, E., and Wagner H. 2016. vegan: Community Ecology Package. R package version 2.4-1. https://cran.r-project.org/

Peay, K. G., Baraloto, C., and Fine, P. V. A. 2013. Strong coupling of plant and fungal community structure across western Amazonian rainforests. ISME J. 7: 1852-1861.

R Development Core Team. 2015. R: A Language and Environment for Statistical Computing. R Foundation for Statistical Computing, Vienna, Austria.

Regalado, V., Rodriguez, A., Perestelo, F., Carnicero, A., De La Fuente, G., and Falcon, M. A. 1997. Lignin degradation and modification by the soilinhabiting fungus Fusarium proliferatum. Appl. Environ. Microbiol. 63: 3716-3718.

Revillini, D., Gehring, C. A., and Johnson, N. C. 2016. The role of locally adapted mycorrhizas and rhizobacteria in plant-soil feedback systems. Funct. Ecol. 30:1086-1098.

Rodriguez-Carres, M., White, G., Tsuchiya, D., Taga, M., and VanEtten, H. D. 2008. The supernumerary chromosome of Nectria haematococca that carries pea-pathogenicity-related genes also carries a trait for rhizosphere competitiveness. Appl. Environ. Microbiol. 74:3849-3856.
Saunders, M., Glenn, A. E., and Kohn, L. M. 2010. Exploring the evolutionary ecology of fungal endophytes in agricultural systems: Using functional traits to reveal mechanisms in community processes. Evol. Appl. 3:525-537.

Scherling, C., Roscher, C., Giavalisco, P., Schulze, E. D., and Weckwerth, W. 2010. Metabolomics unravel contrasting effects of biodiversity on the performance of individual plant species. PLoS One 5:e12569.

Schlatter, D. C., DavelosBaines, A. L., Xiao, K., and Kinkel, L. 2013. Resource use of soilborne Streptomyces varies with location, phylogeny, and nitrogen amendment. Microbiol. Ecol. 66:961-971.

Schlatter, D. C., Bakker, M. G., Bradeen, J. M., and Kinkel, L. 2015. Plant community richness and microbial interactions structure bacterial communities in soil. Ecology 96:134-142.

Steinberg, C., Lecomte, C., Alabouvette, C., and Edel-Hermann, V. 2016. Root interactions with nonpathogenic Fusarium oxysporum: Hey Fusarium oxysporum, what do you do in life when you do not infect a plant? Pages 281-299 in: Belowground Defense Strategies in Plants: Signaling and Communication in Plants. C. Vos and K. Kazan, eds. Springer, Switzerland. Tamura, K., Stecher, G., Peterson, D., Filipski, A., and Kumar, S. 2013. MEGA6: Molecular evolutionary genetics analysis version 6.0. Mol. Biol. Evol. 30:2725-2729.

Tilman, D., Knops, J., Wedin, D., Reich, P., Ritchie, M., and Siemann, E. 1997. The influence of functional diversity and composition on ecosystem processes. Science 277:1300-1302.

Tsavkelova, E., Oeser, B., Oren-Young, L., Israeli, M., Sasson, Y., Tudzynski, B., and Sharon, A. 2012. Identification and functional characterization of indole-3-acetamide-mediated IAA biosynthesis in plant-associated Fusarium species. Fungal Genet. Biol. 49:48-57.

Van Nuland, M. E., Wooliver, R. C., Pfennigwerth, A. A., Read, Q. D., Ware, I. M., Mueller, L., Fordyce, J. A., Schweitzer, J. A., and Bailey, J. K. 2016. Plant-soil feedbacks: Connecting ecosystem ecology and evolution. Funct. Ecol. 30:1032-1042.

van Rij, E. T., Girard, G., Lugtenberg, B. J. J., and Bloemberg, G. V. 2005. Influence of fusaric acid on phenazine-1-carboxamide synthesis and gene expression of Pseudomonas chlororaphis strain PCL1391. Microbiol.-SGM 151:2805-2814.

Wei, T. 2013. corrplot: Visualization of a correlation matrix. R package version 0.73. https://cran.r-project.org/web/packages/corrplot/corrplot.pdf

Weidner, S., Koller, R., Latz, E., Kowalchuck, G., Bonkowski, M., Scheu, S., and Jousset, A. 2015. Bacterial diversity amplifies nutrient based plant-soil feedbacks. Funct. Ecol. 29:1341-1349.

Wickham, H. 2009. ggplot2: Elegant Graphics for Data Analysis. Springer, New York.

Yli-Mattila, T., Kalko, G., Hannukkala, A., Paavanen-Huhtala, S., and Hakala, K. 2010. Prevalence, species composition, genetic variation and pathogenicity of clover rot (Sclerotinia trifoliorum) and Fusarium spp. in red clover in Finland. Eur. J. Plant Pathol. 126:13-27.

Zhao, Z., Liu, H., Wang, C., and Xu, J. R. 2013. Comparative analysis of fungal genomes reveals different plant cell wall degrading capacity in fungi. BMC Genomics 14:274. 